\title{
Innovación en el desarrollo de unidades de producción agrícola familiar en localidades marginadas de Oaxaca
}

\section{Innovation in the development of family agricultural production units in marginalized localities from Oaxaca}

\author{
Domingo Luis Diaz García \\ Instituto Tecnológico de Oaxaca, México \\ zaidyfamily@hotmail.com \\ (D) http://orcid.org/0000-0002-5992-9995 \\ Gerardo Rodriguez Ortiz \\ Instituto Tecnológico del Valle de Oaxaca, México \\ geraro65@gmail.com \\ (i) http://orcid.org/0000-0003-0963-8046 \\ Blasa Celerina Cruz Cabrera \\ Instituto Tecnológico de Oaxaca, México \\ cabreracruz85@hotmail.com \\ (D) http://orcid.org/0000-0003-4694-4261 \\ Maricela Castillo Leal \\ Instituto Tecnológico de Oaxaca, México \\ maricelacastillo3@gmail.com \\ (i) http://orcid.org/0000-0002-3281-4135 \\ Gisela Margarita Santiago Martinez \\ Instituto Tecnológico del Valle de Oaxaca, México \\ gissant68@hotmail.com \\ (D) http://orcid.org/0000-0002-0064-7010
}

Recepción: 19 de febrero de 2019

Aprobación: 20 de febrero de 2020

\section{RESUMEN}

Se estudian los factores de innovación y su incidencia en el desarrollo de unidades de producción agrícola familiar (UPAF) de cultivos de maíz, frijol y jitomate en localidades marginadas de Ejutla de Crespo, en Valles Centrales de Oaxaca. Se analizan con un proceso metodológico mixto no experimental mediante muestreo estratificado-aleatorio por medio del cuestionario $(n=86)$ realizado en 2017 y examinado mediante pruebas de bondad de ajuste $\chi^{2}(\alpha=0.05)$. El enfoque utilizado es evolucionista y sistémico, además de considerar teóricos neochumpeterianos. Los tipos de innovación están relacionados con el cultivo y son independientes de la localidad. De los factores considerados, 9 de 10 inciden en algún tipo de innovación. Las innovaciones se dan principalmente en las UPAF con jitomate en invernadero.

Palabras Clave: cultivos anuales, metodología mixta, teoría evolucionista y sistémica.

\begin{abstract}
The objective was to analyze the factors of innovation and their impact on the development of family agricultural production units (FAPU) of corn, beans and tomatoes crops in marginalized localities of Ejutla de Crespo in the Central Valleys of Oaxaca. The innovation was analyzed with a mixed non-experimental methodological process, using stratified-random sampling, by means of the questionnaire $(n=86)$ applied in 2017 and analyzed by means of goodness-of-fit tests $\chi^{2}(\alpha=0.05)$. The approach is systemic and evolutionary, with neochumpeterian theories. The results show the different innovation types, and how they are related to the crop and are independent of the locality. Nine out of ten factors influence some type of innovation. There is a better interaction of factors to innovate in the FAPU where the main crop is tomato in greenhouse.
\end{abstract}

KEYWORDS: Annual crops, mixed methodology, evolutionary and systemic theory. 


\section{INTRODUCCIÓN}

En un mundo que cambia con rapidez es fundamental innovar constantemente. Por ejemplo, la población rural pobre lo hace cada día: su supervivencia depende de ello. Pero si no cuentan con las capacidades, los activos y el acceso necesarios para los mercados, la información, las redes y los conocimientos, la posibilidad de llevarlo a cabo de una manera que les permita salir de la pobreza es escasa. Puesto que las innovaciones se han dirigido en especial a las personas que representan a un mercado comercial, los pobres de las zonas rurales son quienes más probabilidades tienen de quedar excluidos de estos procesos, aunque actualmente también pueden presentarse en diferentes contextos y consideran no nada más aspectos tecnológicos, sino además de mercado y organizacionales (French et al., 2014).

El medio rural mexicano muestra características similares al de muchos países de América Latina, es decir, está signado por el desequilibrio social y económico, alto nivel de pobreza, bajo nivel de sostenibilidad de la producción y baja productividad agrícola. Este contexto se presenta más aún en las comunidades marginadas, donde la producción agrícola en cultivos anuales es considerada como medio de subsistencia y no para mejorar el nivel socioeconómico (Herrera, 2013; Rendón y Aguilar, 2013). Este tipo de medio se caracteriza por ubicarse en regiones con bajo nivel educativo y baja densidad poblacional, con atributos agroecológicos deficientes, poca conectividad a mercados y baja coordinación institucional. La situación del medio rural del estado de Oaxaca y municipios de la región de los Valles Centrales no es ajena. En este sentido, el área de estudio comprende localidades del municipio de Ejutla de Crespo.

Ante tal realidad, es necesario que en los cambios actuales se busquen estrategias y políticas diferenciadas que permitan atender o hacer partícipe a la población rural pobre para reducir en un primer momento sus condiciones de marginación y pobreza (Berdegué, 2005; FAO, 2012a; Sonnino y Ruane, 2013). Por otro lado, se planteó un sustento teórico que fundamente las características de la agricultura familiar en comunidades marginadas y la forma de cómo éstas pueden formar parte en las diferentes estrategias para mejorar sus condiciones productivas a través de la implementación de innovaciones al conocer los factores que inciden, bajo un enfoque de sistemas, con la participación de las UPAF (Águila y Padilla, 2010).

De acuerdo con FAO (2012a) y Villalobos et al. (2015), las oportunidades y condiciones de la innovación están distribuidas desigual y desfavorablemente en el medio rural, como muchos de los activos y recursos, por lo que esta situación llevó a indagar cuáles son los factores que dan la posibilidad de habilitar a los pobres para ser partícipes en ellas y cambien sus condiciones a través de la diversificación agrícola en cultivos anuales, la organización y la mejora en la productividad (Berdegué, 2005; Rodríguez et al., 2013).

Acorde con el Consejo Nacional de Evaluación de la Política de Desarrollo Social (CONEVAL, 2012), el mayor porcentaje de la población en condiciones de pobreza en México se localiza en Chiapas (75\%), Guerrero (70\%) y Oaxaca (62\%), lugares donde prevalece la pobreza extrema y donde la agricultura es la principal actividad económica de la población rural pobre. Es claro que se necesitan innovaciones favorables que le permitan a este sector aumentar su capital social y aprovechar las oportunidades que les ofrecen las relaciones basadas en el mercado en los ámbitos rural y agrícola, así como cambios tecnológicos para desarrollar y adoptar nuevos conocimientos a fin de que puedan participar en su desarrollo (French et al., 2014; ONU, 2014).

Fue Schumpeter quien teorizó por primera vez este concepto a inicios del siglo XX y subrayó la importancia de la tecnología y el cambio tecnológico mediante las innovaciones, (Montoya, 2004; Chirinos, 2011).

Por el enfoque de este artículo, se ajusta de mejor forma la descripción de Berdegué (2005: 4), quien menciona que las innovaciones "son ideaciones sociales y, por consiguiente, reflejan la interrelación de diferentes agentes -y son resultante de ella-, que a menudo tienen intereses y objetivos contradictorios y, desde luego, distintos grados de poder económico, social y político”. Asimismo, sustenta su enfoque en la teoría de Niels Röling y Engels sobre sistemas, cuyo principio es la cooperación y el aprendizaje (FAO, 2012a) sin perder de vista lo referido en el Manual de Oslo al indicar que la innovación es la implementación de una novedad o mejora para un lugar 
determinado, sea o no tecnológica, en productos, procesos, formas de mercado o de organizarse y tienen lugar cuando existe una apropiación social (French et al., 2014) y puede ser dentro de un proceso económico, social o de impacto ecológico (Aguilar et al., 2017)

De acuerdo con Formichella (2005) y Morales et al. (2014), la evolución del concepto, a partir de su teorización, se resume en cuatro etapas: a) la del empresario innovador como agente aislado, y corresponde al economista Joseph Schumpeter. $b$ ) En un segundo momento el modelo lineal: el proceso de innovación es secuencial, pues comienza con la generación de ideas, investigación, implementación y termina con la difusión (Quintero y Cortés, 2011; Morales et al., 2014).

Las dos fases siguientes, $c$ ) la de gestión de la innovación y d) la de sistemas de innovación, se ubican dentro del pensamiento del aprendizaje interactivo y evolucionista o neoschumpeteriano, que está representado principalmente por Nelson, Winter y Giovanni Dosi (Morales et al., 2014) y para el medio rural por Niels Röling y Engel.

Es difícil establecer un proceso generalizado de implementación que además reúna la complejidad del sistema dentro del cual se desarrollan las innovaciones; sin embargo, se ha establecido que se pueden agrupar en internos u organizacionales y externos, lo cual comprende organizaciones e instituciones (Rugeles et al., 2013; Morales et al., 2014).

La variable teórica innovación para su análisis se dimensiona de tres formas: a) por tipos de innovación: de proceso, de producto, organizacionales y de mercado (OCDE, 2005; French et al., 2014), b) etapas de desarrollo y c) por los factores que inciden en la innovación (Águila y Padilla, 2010). En este caso, se dimensiona desde los factores e interacción de éstos y, como refieren Bisang et al. (2015), esa interacción es determinante para innovar y considerarse como un sistema o procesos interactivos.

Para el sector agropecuario no se puede decir que exista un modelo de innovación único, pero existen elementos que ayudan a entender las interacciones en un sistema, entre los cuales se pueden mencionar: nivel educativo, experiencia del productor, perfil del productor, recurso humano y formación, cambios generados o innovaciones específicas, ambiente de innovación, vínculos locales y/o regionales, investigación y desarrollo, articulación al mercado, participación en redes sociales, considerados dentro de un ámbito local y/o regional, en el que el actor principal, es el productor o las unidades de producción de una determinada localidad o región (FAO, 2012a; Rugeles, 2014).

De acuerdo con lo expuesto, el objetivo es identificar y analizar los factores de la innovación y su incidencia en el desarrollo de las UPAF de localidades en condiciones de marginación de la región de los Valles Centrales de Oaxaca con cultivos de maíz, frijol y jitomate. A partir de este análisis, se pretende conocer las oportunidades del medio rural marginado en agricultura. La hipótesis planteada es que existen factores internos y externos que interactúan y determinan la presencia de innovaciones en las UPAF del medio rural.

\section{Metodología}

\section{1. Área de estudio}

El estudio se realizó en las localidades de Monte del Toro, La Noria de Ortiz y Santa Martha Chichihualtepec, del municipio de Ejutla de Crespo de la región de los Valles Centrales, Oaxaca; la principal actividad es la agricultura de tipo familiar con cultivos de maíz, frijol y jitomate en invernadero.

\subsection{Diseño y tipo de la investigación}

$\mathrm{Al}$ retomar la variable teórica considerada para la presente investigación, y las bases teóricas de ésta, se propone el modelo teórico de innovación (figura 1) con enfoque de sistemas y sustento teórico de procesos interactivos continuos entre los distintos actores y elementos que participan en la del medio rural, en cuyos principios está el aprendizaje y cooperación (Delgado y Escobar, 2009; Morales et al., 2014; French et al., 2014). Este modelo teórico se ubica dentro de la teoría evolucionista, representada entre otros por Nelson, Winter y Dosi. 


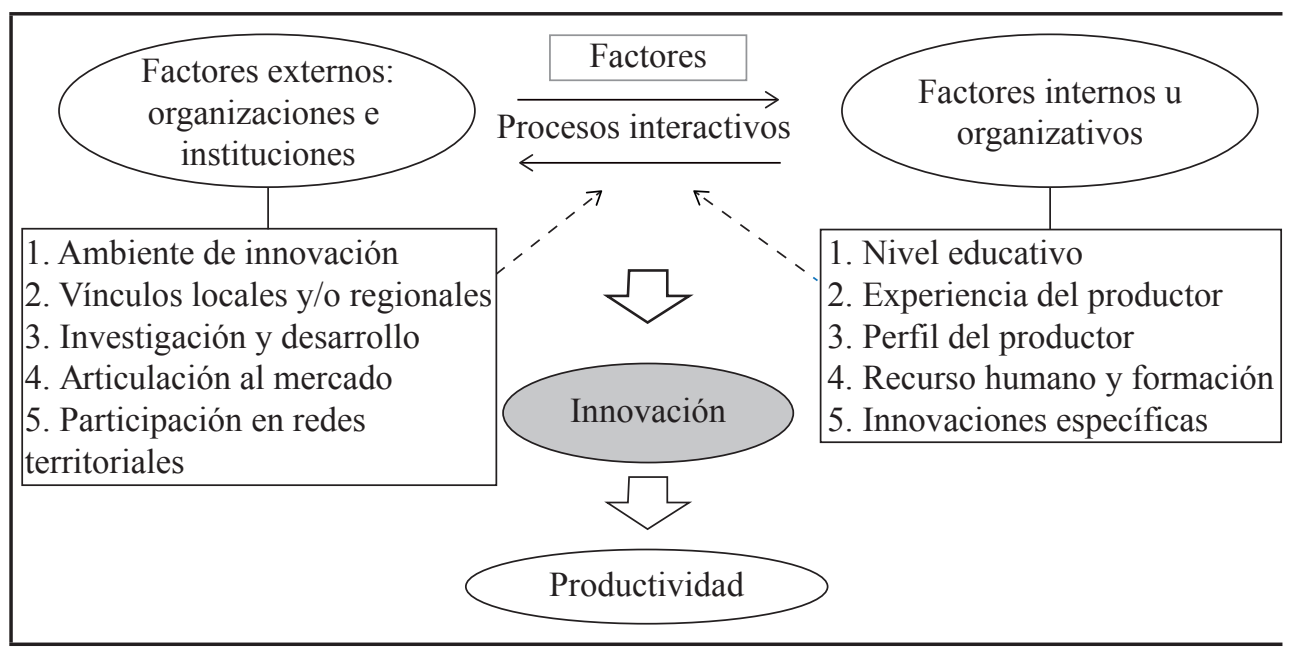

FIGURA 1

Modelo teórico propuesto y utilizado para la elaboración del cuestionario en el proceso metodológico Fuente: elaboración propia con base en Delgado y Escobar (2009); Águila y Padilla (2010); FAO (2012a); Rugeles et al. (2013); Morales et al. (2014).

El diseño de investigación elegido fue de tipo no experimental, pues no hubo manipulación deliberada de la variable y porque se buscó hacer una revisión de la variable teórica innovación. Como lo mencionan Hernández et al. (2014), lo que hace que una investigación sea no experimental es poder observar los fenómenos tal y como se dan en el contexto real para su posterior análisis.

El análisis comprendió un periodo, por lo que el diseño de investigación no experimental es de tipo transeccional o transversal, es decir, se hace un corte en el tiempo. De acuerdo con Hernández et al. (2014), se busca describir variables, analizar su incidencia e interrelación de factores en un momento dado; para ello, se tomaron como referencia investigaciones realizadas en el sector agropecuario por Rugeles et al. (2013) y Rugeles (2014), quienes refieren que un intervalo de dos a cinco años anteriores es suficiente para acotar y poder inferir sin que se alteren los resultados. Se hace alusión al paradigma mixto, es decir, se apoya del enfoque cuantitativo y cualitativo, con mayor uso del cuantitativo, puesto que lleva un proceso más definido al existir un tamaño de muestra, un instrumento estructurado para la colección de información, un procedimiento de aplicación y estadísticos definidos y el segundo enfoque lo complementa con entrevistas y registros de campo menos estructurados (figura 2). Este enfoque se hizo para la cualificación de datos cuantitativos que arrojó el cuestionario.

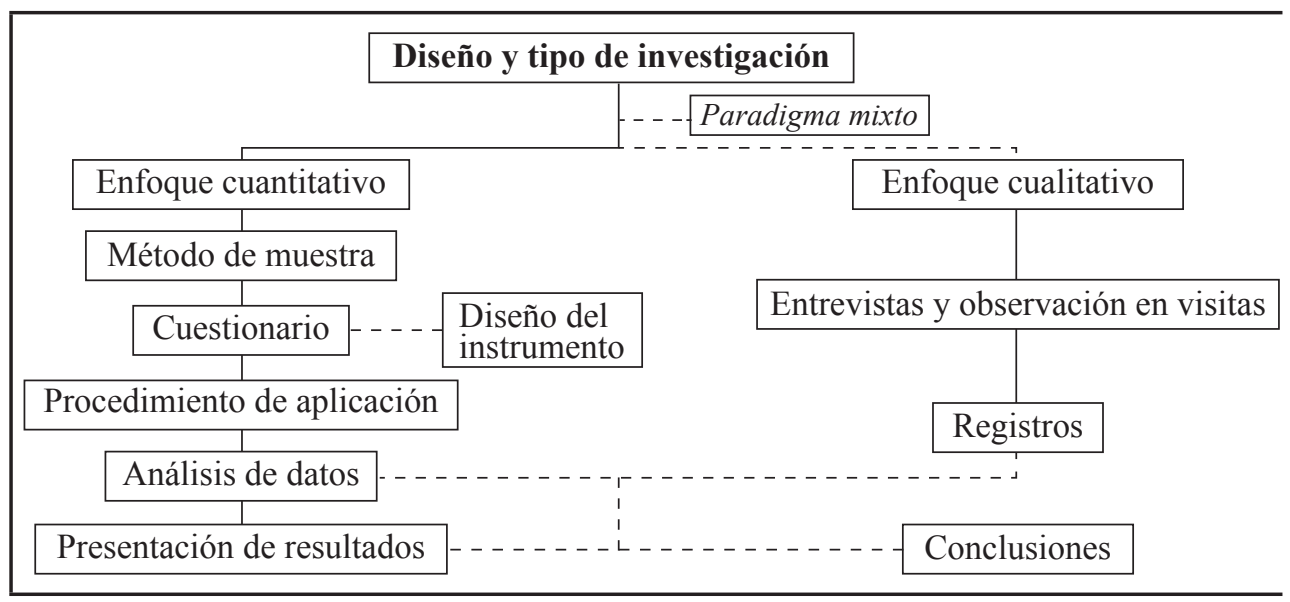

FIGURA 2

Marco metodológico

Fuente: elaboración propia con apoyo del marco teórico de Rojas (2013) y Hernández et al. (2014). 


\subsection{Diseño de muestreo}

Se utilizó muestreo estratificado-aleatorio para proporciones dividiendo las UPAF en tres estratos correspondientes a las tres localidades rurales en condiciones de marginación. Dentro de cada comunidad, las UPAF se seleccionaron aleatoriamente de manera proporcional al tamaño de la población objetivo (Díaz et al., 2016). Las unidades de muestreo fueron las UPAF con cultivos de maíz, frijol y jitomate, este último en condiciones de invernadero.

Las UPAF son consideradas unidades económicas de producción sometidas a una gerencia única que poseen acceso limitado a recursos de tierra y capital y uso preponderante de fuerza de trabajo familiar, siendo el jefe de familia quien participa de forma directa en el proceso de producción y quien no asume funciones exclusivas de gerente, sino que es un trabajador más del núcleo familiar (FAO, 2012b; De la O y Guzmán, 2014).

Para el cálculo del tamaño de muestra se tomó en cuenta que la población se distribuye en grupos (estratos) para reducir el error y se utiliza un cuestionario con demasiadas preguntas (Rojas, 2013), por lo que se empleó la fórmula para una muestra estratificada (Scheaffer et al., 2007).

$$
n=\frac{\sum_{i=1}^{L} \frac{\left(N_{i}^{2} p_{i} q_{i}\right)}{w_{i}}}{N^{2} D+\sum_{i=1}^{L} N_{i} p_{i} q_{i}}
$$

Donde: $n$ tamaño de muestra (86), $N$ población objeto de estudio (717), Z95\% nivel de confianza, $E$ precisión con la que se generalizan los resultados $0.10, p=0.80$ y $q=0.20$ proporción de que sean UPAF y que no lo sean, respectivamente (variabilidad de la población). $N_{i}$ = población objetivo de cada estrato, Santa Martha Chichihualtepec $N_{1}$ : 436 UPAF; Monte del Toro $N_{2}: 178$ UPAF y La Noria de Ortiz $N_{3}: 103$ UPAF.

Se considera que el error de muestreo (EM) está dentro de lo permitido para trabajos en el área social, ya que variaciones mayores al 10\% reducen la validez de la información (Kulshreshtha, 2013), por lo cual no existió la necesidad de incrementar el tamaño de muestra definitivo. El EM fue calculado con base en la proporción de UPAF que producen cultivos objeto de este estudio; el valor de la media estratificada fue $\overline{\mathbf{y}}_{\mathrm{ST}}=49$.42, la varianza de la media estratificada $\operatorname{Var}\left(\overline{\mathrm{Y}}_{\mathrm{ST}}\right)=1.148$, el intervalo de confianza IC $(95 \%)\left(t_{\alpha / 2}\right)^{2}=49.42 \pm 2.14$ y el error muestral EM $=2.3 \%$ (Kulshreshtha, 2013).

\section{4. Instrumento utilizado: el cuestionario y la entrevista}

En la elaboración del cuestionario fue necesario la operacionalización de la variable teórica innovación (Babbie, 2000), por lo que se tomó como referencia el modelo teórico propuesto, mismo que se fundamentó en el enfoque evolucionista de procesos interactivos; la variable de innovación comprendió dos dimensiones (cada una con sus respectivos indicadores): la primera de factores internos y la segunda de factores externos. El proceso de operacionalización consistió en pasar de lo abstracto (variable teórica) a lo concreto (variables medibles), es decir, definir los indicadores que ayudaron a la interpretación de la variable teórica. Con este proceso se determinó el método a través del cual la variable fue medida o analizada.

La variable teórica se desglosó a través de un proceso de deducción lógica, en indicadores que representan ámbitos específicos de la variable. Los indicadores a su vez se midieron por medio de items o preguntas que se incluyeron en el cuestionario. A este proceso de operacionalización se le conoce también como deducción de consecuencias verificables, ya que fueron las relaciones entre los indicadores los que se sometieron a verificación empírica (Rojas, 2013). Es así como la variable innovación, una vez operacionalizada, contempló 10 indicadores (figura 1). 
Estos 10 indicadores formaron parte del análisis cuantitativo y se apoyó en el análisis cualitativo, este último por medio de entrevistas a autoridades agrarias, municipales y profesionistas del sector para fortalecer la discusión y las conclusiones, haciendo referencia al paradigma mixto. Los indicadores considerados fueron, en la dimensión de factores internos, el nivel educativo, edad y experiencia del productor, perfil del productor, recurso humano y formación y cambios generados o innovaciones específicas; en la dimensión de factores externos, el ambiente de innovación, vínculos locales y/o regionales, investigación y desarrollo, articulación al mercado y participación en redes territoriales (figura 1).

El método empleado para el análisis cuantitativo, es decir el cómo, fue a través de muestreo probabilístico de la población, la técnica empleada, la encuesta (a través de qué) y el instrumento utilizado (con qué) fue el cuestionario (Rojas, 2013). Es decir, se utilizó la técnica de la encuesta estructurada, con el uso de un cuestionario, que se aplicó a productores responsables de las UPAF seleccionadas o consideradas en el muestreo. El instrumento contempló dos secciones: la primera de información general con 14 preguntas, la segunda, y más amplia, consideró información de la variable innovación. Esta última se dividió a su vez en dos partes: la primera que correspondió a la dimensión de factores internos con cinco indicadores y la segunda a la dimensión de factores externos con cinco indicadores; la segunda sección se integró con preguntas tipo Likert, con cinco alternativas de respuesta (Babbie, 2000), el cuestionario comprendió 84 preguntas.

Antes de aplicar el cuestionario definitivo, se llevó a cabo la revisión de estructura y redacción para verificar su calidad; posteriormente, una prueba piloto para comprobar la confiabilidad y la validez del instrumento. Esta prueba se efectuó de manera aleatoria y en forma presencial con 20 productores de las tres localidades del municipio objeto de estudio. En esta fase se reestructuraron items que no explicaban o guardaban relación alguna con el indicador a través de un análisis de factores; esta fase fue importante en la definición del cuestionario. La aplicación del cuestionario en sus dos fases se hizo de forma presencial cara a cara con los productores.

\section{5. Procesamiento y análisis de la información}

Antes de realizar la prueba fue necesario generar una base de datos en Excel® y realizar la depuración de datos, especialmente la "no respuesta" encontrada en los cuestionarios. El cuestionario fue codificado con la finalidad de hacer más rápida su captura y facilitar su análisis a través del apoyo del paquete estadístico Statistical Analysis System ${ }^{\circledR}($ SAS) (Pérez, 2010).

Se efectuaron pruebas de bondad de ajuste mediante Ji-cuadrada $\left(\chi^{2}, 0.05\right)$ para probar independencia de variables (Levin et al., 2004). Con esta prueba se detectaron las variables significativas en lo relacionado con los factores de la innovación, con respecto a las UPAF de maíz, frijol y de jitomate y a cada una de las localidades consideradas. Esto se complementó con información obtenida de las entrevistas a autoridades municipales y ejidales y a profesionistas del sector y de los registros de las observaciones en las visitas.

\section{Resultados y discusión}

\section{1. Factores internos y externos en relación con la edad del productor}

De la variable considerada en el modelo teórico y operacionalizada para su análisis, dentro de la dimensión de factores internos, la edad del productor resultó independiente de las innovaciones $\left(5.90^{\text {ns }} p>0.05\right)$ que se generan en las UPAF (cuadro 1). Es decir, la edad de los productores de las UPAF no es un factor influyente para realizar procesos de innovación en cada uno de los cultivos indicados, situación observada para la dimensión de factores externos (cuadro 1), realidad que no coincide con lo reportado por Rugeles (2014) para unidades de producción agrícola en Colombia, donde la edad resultó ser determinante. Sin embargo, 
los resultados obtenidos en la presente investigación se justifican, ya que para innovar se requiere voluntad y acción que no necesariamente tiene que ver con la edad (Valencia y Patlan, 2011).

Lo anterior se explica con lo referido por los mismos productores, esto es, que en ellos se da la cooperación y apoyo en las diferentes actividades agrícolas y de este modo alcanzar nuevos aprendizajes, como lo refiere Röling y Engel, en la referencia de Berdegué (2005) y la FAO (2012a). Los factores internos y externos que interactúan para propiciar innovaciones de diferentes tipos resultan no significativos cuando se relacionan con la edad del productor, al menos en lo que respecta a localidades en condiciones de marginación en donde se practica una agricultura de tipo familiar. En este caso se observa que únicamente el nivel educativo tiene una alta dependencia con la edad del productor aun cuando el promedio de escolaridad para estas comunidades rurales es de 5.3 años, y que incide para que el estudio se desarrolle en centros de investigación (cuadro 1).

\section{CUADRO 1}

Dependencia de factores internos y externos de innovación con relación a la edad del productor

\begin{tabular}{|c|c|c|c|}
\hline \multicolumn{4}{|c|}{ Edad del productor } \\
\hline Factores internos & $\chi^{2}$ & Factores externos & $\chi^{2}$ \\
\hline Escolaridad del productor & & Ambiente de innovación & \\
\hline Nivel educativo formal & $21.98^{* *}$ & El desarrollo de actividades & $7.24^{\mathrm{ns}}$ \\
\hline Experiencia del productor & & significativamente nuevas & \\
\hline Tiempo de ser productor y rendimientos obtenidos & $11.78^{\mathrm{ns}}$ & Vínculos locales y/o regionales & \\
\hline Perfil del productor & $7.71 \mathrm{~ns}$ & Con instituciones para recibir & $4.71^{\mathrm{ns}}$ \\
\hline $\begin{array}{l}\text { Forma parte de una sociedad de producción rural } \\
\text { Opera como UPAF }\end{array}$ & $1.06^{\mathrm{ns}}$ & $\begin{array}{l}\text { asistencia técnica y capacitación } \\
\text { Investigación } \mathbf{y} \text { desarrollo }\end{array}$ & \\
\hline Recurso humano y formación & $5.70^{\mathrm{ns}}$ & La investigación se realiza en centros & $13.72^{*}$ \\
\hline Trabajadores familiares & $11.57^{\mathrm{ns}}$ & de investigación & \\
\hline Capacitación relacionada con el proceso de & & Articulación al mercado & \\
\hline producción & & La venta se hace en la localidad y/o en & $5.47^{\mathrm{ns}}$ \\
\hline Cambios generados o innovaciones específicas de: & & el mercado regional & \\
\hline Proceso & $6.32^{\mathrm{ns}}$ & Participación en redes territoriales & \\
\hline Manejo del cultivo & & Coordinación con centros de & $1.61^{\mathrm{ns}}$ \\
\hline Producto & $1.71^{\mathrm{ns}}$ & investigación y desarrollo & \\
\hline Producto de una variedad diferente & & Crear organizaciones productivas & $3.91^{\mathrm{ns}}$ \\
\hline Organización & $2.99 \mathrm{~ns}$ & & \\
\hline $\begin{array}{l}\text { Contratación personal de acuerdo con sus } \\
\text { conocimientos }\end{array}$ & $5.20^{\mathrm{ns}}$ & & \\
\hline Coordinación con otros productores agrícolas & & & \\
\hline Mercado & $7.65^{\mathrm{ns}}$ & & \\
\hline $\begin{array}{l}\text { La comercialización se hace durante todo el año } \\
\text { Del mercado regional al estatal }\end{array}$ & $3.82^{\mathrm{ns}}$ & & \\
\hline
\end{tabular}

Nota: prueba de independencia de bondad y ajuste $\left(\chi^{2}, \alpha=0.05\right) .{ }^{*}, *$ Significativo $=p \leq 0.05$ y $p \leq 0.01$, respectivamente, ns $=$ no significativo.

\section{2. Factores internos y externos en relación con la unidad de producción agrícola familiar (UPAF)}

Respecto a la comparación que se hace entre el tipo de UPAF y las diferentes variables de la innovación, se observa que existe, en la mayoría de las variables de innovación una fuerte dependencia con el tipo de cultivo $(p<0.01)$ para factores internos u organizativos y para factores externos (cuadro 2); sin embargo, la escolaridad no mostró dependencia con respecto al tipo de UPAF. Esto último se explica para este sector y en específico para localidades consideradas marginadas, donde la experiencia del productor resulta ser más determinante que el nivel educativo, 
el cual generalmente es bajo, como lo menciona Berdegué (2005) y Rugeles et al. (2013). No obstante, cuando el sistema de innovación adquiere mayor complejidad esta variable influye en determinadas innovaciones específicas, como lo refieren Águila y Padilla (2010) y Sonnino y Ruane (2013) cuando mencionan que la educación es parte del triángulo del conocimiento necesario para generar innovaciones, ya que estas son más complejas mientras existan mayores interacciones entre factores, como sucede en este caso con las UPAF con jitomate como cultivo principal, lo que coincide con Vargas et al. (2015) cuando refieren que la innovación se relaciona con difusión de conocimientos.

\section{CUADRO 2}

Dependencia de factores internos y externos de la innovación en relación con el tipo de unidad de producción agrícola familiar (UPAF)

\begin{tabular}{|c|c|c|c|}
\hline \multicolumn{4}{|c|}{ Tipo de UPAF } \\
\hline Factores internos & $\chi^{2}$ & Factores externos & $\chi^{2}$ \\
\hline Escolaridad del productor & \multicolumn{2}{|r|}{ Ambiente de innovación } & \\
\hline Nivel educativo formal & $8.17^{\mathrm{ns}}$ & El desarrollo de actividades significativamente & $35.63^{* *}$ \\
\hline \multicolumn{2}{|l|}{ Experiencia del productor } & nuevas son con ideas del productor & \\
\hline Gestión de apoyos & $23.96^{* *}$ & \multirow{2}{*}{$\begin{array}{l}\text { De instituciones del Gobierno y de } \\
\text { investigación }\end{array}$} & \multirow[t]{3}{*}{$73.06^{* *}$} \\
\hline Tiempo de ser productor y rendimientos & $11.78^{\mathrm{ns}}$ & & \\
\hline obtenidos & $18.54^{* *}$ & \multirow{2}{*}{ Vínculos locales y/o regionales } & \\
\hline Cambios al proceso de producción & $39.81^{* *}$ & & $32.59^{* *}$ \\
\hline \multicolumn{2}{|l|}{ Perfil del productor } & Relación con proveedores & $69.56^{* *}$ \\
\hline Forma parte de una Sociedad de & $22.18^{* *}$ & Relación con instituciones para recibir & \multirow[t]{2}{*}{$54.06^{* *}$} \\
\hline Producción & $30.06^{* *}$ & $\begin{array}{l}\text { asistencia técnica y capacitación } \\
\text { Investigación y desarrollo }\end{array}$ & \\
\hline Rural & & Investigación y desarrollo & \\
\hline Opera como UPAF & & Con recursos propios de la unidad & $13.64^{*}$ \\
\hline \multirow{2}{*}{$\begin{array}{l}\text { Adquisición de insumos de } \\
\text { forma colectiva }\end{array}$} & $13.82^{\mathrm{ns}}$ & \multirow{2}{*}{$\begin{array}{l}\text { Con recursos de terceros } \\
\text { (INIFAP, Fundación Produce) }\end{array}$} & \multirow[t]{2}{*}{$20.34^{* *}$} \\
\hline & $29.27^{* *}$ & & \\
\hline \multirow{5}{*}{$\begin{array}{l}\text { Recurso humano y formación } \\
\text { Trabajadores familiares } \\
\text { Trabajadores no familiares } \\
\text { Capacitación relacionada con el proceso } \\
\text { de producción }\end{array}$} & $66.91^{* *}$ & \multirow{3}{*}{$\begin{array}{l}\text { La investigación se realiza en centros de } \\
\text { investigación } \\
\text { Articulación al mercado }\end{array}$} & \multirow[t]{3}{*}{$28.07^{* *}$} \\
\hline & & & \\
\hline & & & \\
\hline & & La venta se realiza en la localidad & $35.57^{* *}$ \\
\hline & & \multirow{3}{*}{$\begin{array}{l}\text { La venta se hace en la localidad y en el } \\
\text { mercado regional } \\
\text { El total de la venta se hace fuera de la localidad }\end{array}$} & \multirow[t]{2}{*}{$46.09^{*}$} \\
\hline \multirow{2}{*}{$\begin{array}{l}\text { Cambios generados o innovaciones } \\
\text { específicas de: }\end{array}$} & $67.88^{* *}$ & & \\
\hline & $54.79^{* *}$ & & \multirow[t]{2}{*}{$94.12^{* *}$} \\
\hline Proceso & $84.18^{* *}$ & Participación en redes territoriales & \\
\hline Uso de nuevas variedades & & \multirow{2}{*}{$\begin{array}{l}\text { Para emprendimientos en la UPF con otros } \\
\text { productores }\end{array}$} & \multirow{3}{*}{$56.43^{* *}$} \\
\hline Manejo del cultivo & & & \\
\hline Manejo adecuado de agroquímicos & $76.57^{* *}$ & \multirow{2}{*}{$\begin{array}{l}\text { Coordinación con centros de investigación } \\
\mathrm{y} \text { desarrollo }\end{array}$} & \\
\hline Producto & $76.84^{*}$ & & $23.94^{* *}$ \\
\hline $\begin{array}{l}\text { Producto de una variedad diferente } \\
\text { Clasificación del producto }\end{array}$ & $78.23^{* *}$ & Crear organizaciones productivas & $50.96^{* *}$ \\
\hline Organización & $75.02^{* *}$ & & \\
\hline $\begin{array}{l}\text { Contratación personal de acuerdo a sus } \\
\text { conocimientos }\end{array}$ & $14.21^{*}$ & & \\
\hline Se definen tareas & & & \\
\hline Coordinación con otros productores agrícolas & $38.15^{* *}$ & & \\
\hline Mercado & $78.52^{* *}$ & & \\
\hline La comercialización se hace durante todo el año & $20.43^{* *}$ & & \\
\hline Del mercado regional al estatal & & & \\
\hline Se apoya de instituciones & & & \\
\hline
\end{tabular}

Nota: prueba de independencia de bondad y ajuste $\left(\chi^{2}, \alpha=0.05\right), \mathrm{UPAF}=$ Unidad de producción agrícola familiar. ${ }^{*},{ }^{*}$ Significativo $p$ $\leq 0.05$ y $p \leq 0.01$, respectivamente, ${ }^{\mathrm{ns}}=$ no significativo. 
Existe una fuerte dependencia entre las UPAF y los tipos de innovación $(p<0.01)$, y, de acuerdo con Rendón y Aguilar (2013), cuando existen modelos alternativos de adopción de tecnologías se promueven diferentes tipos de innovación con la participación de receptores como son los productores y la vinculación con instituciones, en este caso los procesos interactivos entre factores. El tipo de UPAF determina un mayor grado de interacción entre factores y al mismo tiempo el desarrollo de diferentes innovaciones, por lo que, de acuerdo con el cultivo que se considere como principal, el productor realiza más actividades de organización, mercado y, como consecuencia, procesos de producción diferentes (cuadro 2).

Cabe mencionar que por localidad, al igual como sucede con la edad del productor, no se encuentra dependencia, por lo que para este caso la relación con los factores de la innovación está en función del tipo de cultivo que la UPAF considere como actividad principal, encontrándose innovaciones no diferenciadas por localidad. Lo anterior se explica por ser localidades con características culturales, sociales y geográficamente similares, como se describe en el Plan de Desarrollo del Municipio de Ejutla de Crespo, Oaxaca y, como lo describe la FAO (2012b) y Douwe (2013), en localidades rurales la agricultura es de tipo familiar con diferente grado de consolidación de acuerdo con sus activos y relaciones de cooperación con otras localidades.

De igual forma, se observa que las UPAF tienen mayor interés en interactuar con el entorno para generar ambientes de innovación favorables, pero esto se da en función del cultivo (cuadro 2), ya que los resultados muestran diferentes formas de organización, de interrelación con productores, instituciones, proveedores y clientes, siendo la tendencia de una organización mejor estructurada hacia UPAF con jitomate; esto se valida lo descrito por Arraut (2010) y Britez y Duarte (2013), quienes refieren que la innovación de tipo organizacional es determinante en otra que cuente con efecto positivo sobre la productividad. Con ello se confirma la interacción entre factores internos y externos, necesaria para considerarlos parte de un sistema innovativo, donde no existe linealidad sino interacción de procesos (French et al., 2014).

El nivel de organización encontrado en las diferentes UPAF marca claras diferencias entre ellas, observándose una mejor organización en las que tienen como actividad principal la producción de jitomate, que se articulan más con su entorno e internamente como UPAF, y les genera mayores ingresos permitiéndoles la contratación de mano de obra no familiar, lo cual en cierta forma coincide con Rugeles et al. (2013), quienes mencionan que los resultados encontrados en innovación varían de acuerdo con la cadena productiva. En estas localidades se ve una agricultura familiar entre la transición y consolidación (FAO, 2012b), además de observarse un fenómeno en relación con la mano de obra familiar, la cual resulta insuficiente.

\section{3. Los tipos de unidad de producción agrícola familiar (UPAF) y su tendencia a innovar}

Las UPAF que tienen como cultivo principal al maíz y al frijol prácticamente permanecen sin cambios, rara vez generan innovaciones o se dan de forma aislada; esto se observa en las tres localidades, una situación que no sucede con el cultivo de jitomate en invernadero, donde los productores generan cambios, como se puede observar en los diferentes indicadores de las dos dimensiones consideradas para medir la innovación (cuadro 3). Este escenario en parte coincide con lo encontrado por Rugeles et al. (2013) y Rugeles (2014), cuando hacen alusión a que las innovaciones tienen relación con las cadenas productivas y con el lugar en donde se lleven a cabo; en esto último no hay coincidencia con esta investigación, donde no hay diferencia entre localidades.

Referente a la participación de los productores en el mercado, se observa una marcada relación inversa entre las UPAF de maíz y frijol contra las de jitomate, ya que la tendencia de estas últimas es significativa con un valor de $\chi^{2}=94.13^{* *}$ y $p<0.0001$, con una tendencia hacia una participación mayor a otros mercados, caso contrario con las primeras (cuadro 3). Es importante destacar que aun cuando existen diferencias entre UPAF para participar en mercados distintos al local, $56 \%$ de éstas nunca o rara vez participa en mercados regionales y $36 \%$ lo hace con regularidad o frecuentemente, porcentaje que corresponde a las UPAF de jitomate consideradas en la muestra. 


\section{CUADRO 3}

Relación de las UPAF con su participación en el mercado regional, recurso humano, introducción de variedades y vínculos con productores e instituciones

\begin{tabular}{|c|c|c|c|c|c|c|}
\hline UPAF & Nunca & Rara vez & Regularmente & Frecuentemente & Siempre & Total \\
\hline \multicolumn{7}{|c|}{ Mercado regional $^{* *}$} \\
\hline Frijol & $19(0.22) \uparrow$ & $10(0.12)$ & $1(0.01)$ & & & $30(0.35)$ \\
\hline Maíz & $3(0.03)$ & $16(0.19)$ & $6(0.07)$ & & & $25(0.29)$ \\
\hline Jitomate & & & $6(0.07)$ & $21(0.24)$ & $4(0.05)$ & $31(0.36)$ \\
\hline Total & $22(0.25)$ & $26(0.31)$ & $13(0.15)$ & $21(0.24)$ & $4(0.05)$ & $86(1.00)$ \\
\hline \multicolumn{7}{|c|}{ Recurso humano (capacitación**) } \\
\hline Frijol & $6(0.07)$ & $16(0.19)$ & $3(0.03)$ & & & $25(0.29)$ \\
\hline Maíz & $21(0.24)$ & $9(0.10)$ & & & & $30(0.35)$ \\
\hline Jitomate & $1(0.01)$ & $5(0.06)$ & $17(0.20)$ & $4(0.05)$ & $4(0.05)$ & $31(0.36)$ \\
\hline Total & $28(0.32)$ & $30(0.35)$ & $20(0.23)$ & $4(0.05)$ & $4(0.05)$ & $86(1.00)$ \\
\hline \multicolumn{7}{|c|}{ Introducción de variedades ${ }^{* *}$} \\
\hline Frijol & $6(0.07)^{\oplus}$ & $16(0.19)$ & $3(0.03)$ & & & $25(0.29)$ \\
\hline Maíz & $21(0.24)$ & $9(0.10)$ & & & & $30(0.35)$ \\
\hline Jitomate & $1(0.01)$ & $5(0.06)$ & $17(0.20)$ & $4(0.05)$ & $4(0.05)$ & $31(0.36)$ \\
\hline Total & $28(0.32)$ & $30(0.35)$ & $20(0.23)$ & $4(0.05)$ & $4(0.05)$ & $86(1.00)$ \\
\hline \multicolumn{7}{|c|}{ Vínculos con productores e instituciones** } \\
\hline Frijol & & $8(0.09)^{\bullet}$ & $13(0.15)$ & $3(0.03)$ & $1(0.01)$ & $25(0.29)$ \\
\hline Maíz & $6(0.07)$ & $15(0.17)$ & $9(0.10)$ & & & $30(0.35)$ \\
\hline Jitomate & & $2(0.02)$ & $23(0.27)$ & $5(0.06)$ & $1(0.01)$ & $31(0.36)$ \\
\hline Total & $6(0.07)$ & $25(0.29)$ & $45(0.52)$ & $8(0.09)$ & $2(0.02)$ & $86(1.00)$ \\
\hline
\end{tabular}

Nota: IFrecuencia absoluta y relativa en una muestra $n=86$. Prueba de independencia de bondad de ajuste $\left(\chi^{2}, \alpha=0.05\right)$. ${ }^{* *}=$ Valores con alta significancia $p<0.01$.

Otra variable que muestra una relación o dependencia muy fuerte con el tipo de cultivo es la formación o capacitación del recurso humano con un valor de $\chi^{2}=66.91^{* *}, p<0.0001$, ya que los productores refieren que para el cultivo de maíz y frijol prácticamente no la necesitan, y para el cultivo de jitomate es una necesidad, por lo que hacen uso de ella con más regularidad. Del total de las UPAF, 24\% manifiesta no necesitar capacitación, que corresponde a $70 \%$ de las unidades con cultivo de maíz, caso contrario sucede con las UPAF de jitomate, donde $68 \%$ hace regularmente este proceso (cuadro 3). Esto conlleva a que el productor interactúe cada vez más con su entorno, es decir, con otros productores, instituciones y agentes del sector.

Dentro de las innovaciones específicas observadas, y que tienen que ver con innovaciones de proceso, producto y mercado, está el uso de variedades diferentes. Como en los casos anteriores, se muestra una fuerte dependencia de las innovaciones con el tipo de UPAF $\left(67.88^{* *}\right)$; del total de las UPAF, $67 \%$ nunca o rara vez hace innovaciones, como es en este caso con la introducción de variedades, y son las unidades con jitomate las que innovan, ya que del total de UPAF de jitomate $68 \%$ hace uso de variedades diferentes; caso contrario sucede en los cultivos de frijol o maíz, donde rara vez lo hacen, situación observada en las tres localidades que formaron parte del estudio e independientemente de la edad del productor (cuadro 3).

Los resultados hacen alusión a factores organizacionales inherentes a las unidades de producción agrícola familiar (cuadro 3), en donde se observa una tendencia a buscar el cambio en unidades cuyo cultivo principal es 
el jitomate $\left(\chi^{2}=54.06^{* *}, p<0.0001\right)$. Estos cambios generalmente dependen de su relación con el entorno, es decir, con instituciones y organizaciones (cuadro 3), donde la mayor proporción de UPAF que interactúan más frecuentemente con su entorno son las que tienen como actividad principal el cultivo de jitomate, es decir, 34\% del total, mismas que representan 94\% de las UPAF de jitomate. Esta circunstancia se observa en las variables de investigación, mercado y participación en redes locales para el emprendimiento en actividades inherentes al proceso de producción. Como lo refiere Rugeles et al. (2013), el comportamiento de la innovación resulta diferente principalmente entre cultivos.

\section{Conclusiones}

En conformidad con el modelo teórico propuesto y a los resultados, se concluye que 9 de los 10 factores considerados son determinantes para que existan innovaciones en las unidades de producción agrícola familiar (UPAF), siendo la edad del productor un factor poco influyente, al menos para las comunidades rurales de los Valles Centrales de Oaxaca.

Existen factores internos u organizativos como externos que influyen en que las UPAF innoven, y su grado de interacción depende del tipo de cultivo que considera la UPAF como principal (comprobación de la hipótesis). En el caso de este trabajo, las UPAF con cultivo de jitomate en invernadero son las que tienen más interacciones entre factores y en consecuencia más innovaciones específicas.

Los procesos de innovación en comunidades rurales del municipio de Ejutla de Crespo, Oaxaca, se dan con base en el tipo de cultivo que considera la UPAF como principal y no entre localidades que consideran unidades de producción similares.

Los productores están más interesados en realizar innovaciones cuando se trata de cultivos cuyo destino principal de la producción no es para el autoconsumo sino un mercado, en este caso las UPAF con jitomate.

Los productores de jitomate en condiciones de invernadero, aun cuando tienen menos tiempo tienen de cultivar respecto al cultivo de maíz y frijol, son los que tienen esquemas de producción, comercialización y organización más definidos.

Las principales innovaciones específicas encontradas fueron en el cultivo de jitomate, las cuales consistieron en cambio de variedades, sistemas de riego diferentes, uso de mano de obra ya no familiar en la cual existen asignación de tareas específicas (organización interna de la UPAF), clasificación del producto para acceder a nuevos mercados, formas de organización entre UPAF, que es para solventar sus necesidades de capacitación, asistencia técnica (tan necesaria por el bajo nivel educativo formal observado), adquisición de insumos y su relación con el entorno.

\section{ANálisis PRospectivo}

La visión que tiene este artículo es la de contribuir al desarrollo rural de comunidades en condiciones de marginación. De este modo, con los resultados obtenidos se da evidencia de que los productores del medio rural marginado, a través de diferentes tipos de innovaciones en su actividad agrícola, mejoran sus condiciones socioeconómicas, pero es claro que el bajo nivel educativo que tienen hace que dependan de factores externos para solventar estas deficiencias, situación que los ha llevado a definir un perfil, el cual contempla como factor principal la organización entre las UPAF. Entre las posibles estrategias para dinamizar los procesos de interacción entre diferentes agentes que intervienen en la innovación que se observan en las UPAF está la de reducir sus debilidades mediante un sistema de extensionismo, propio para estas comunidades, que sirva de puente entre las UPAF y su entorno (organizaciones e instituciones) y fortalezca el proceso de retroalimentación continuo para incentivar innovaciones que mejoren las condiciones de los productores. 


\section{Agradecimientos}

Se agradecen los comentarios de los árbitros de la revista que mejoraron sustancialmente el contenido del artículo.

\section{REFERENCIAS}

Águila, A. y Padilla, A. (2010). Factores determinantes de la innovación en empresas de economía social. La importancia de la formación y de la actitud estratégica. CIRIEC-España. Revista de economía pública, socialy cooperativa, 67, 129-155. Disponible en http://www.ciriec-revistaeconomia.es/banco/6706_Aguila_y_Padilla.pdf

Aguilar, G. N., Olvera, M. J., Martínez, G. E., Aguilar, A. J., Muñoz, R. M. y Santoyo, C. H. (2017). La intervención en red para catalizar la innovación agrícola. Revista Hispana para el Análisis de Redes Sociales, 28(1). Disponible en http://dx.doi.org/10.5565/rev/redes.653

Arraut, C. L. (2010). La gestión de calidad como innovación organizacional para la productividad en la empresa. Revista EAN.

Babbie, E. (2000). Fundamentos de la investigación social. México: Internacional Thomson Editores.

Berdegué, J. (2005). Sistemas de innovación favorables a los pobres. Documento de antecedentes presentado al $29^{\circ} \mathrm{Pe}-$ riodo de sesiones del Consejo de Gobernadores del FIDA. Fondo Internacional de Desarrollo Agrícola (FIDA).

Bisang, R., Anlló, G. y Campi, M. (2015). Políticas tecnológicas para la innovación: la producción agrícola argentina. Santiago: CAF. CIEPLAN.

Britez, C. y Duarte, M. (2013). El proceso de innovación en empresas familiares de la zona T del Paraguay. Revista Internacional de Investigación en Ciencias Sociales, 9(1), 75-96.

Chirinos, J. M. (2011). Perspectiva teórica de la innovación organizacional y la gestión del talento humano en las empresas públicas del estado de Zulia, Venezuela. Revecitec URBE, 2(1).

CONEVAL. (2012). Resultados de pobreza en México a nivel nacional y por entidades federativas. Disponible en http://www.coneval.gob.mx

De la O, A. y Guzmán, L. (2014). El concepto de agricultura familiar en América Latina y el Caribe. S. Salcedo y L. Guzmán (eds.), Agricultura familiar en América Latina y el Caribe: recomendaciones de politica. Santiago: FAO.

Delgado, B. y Escobar, B. (2009). Innovación tecnológica, soberanía y seguridad alimentaria. La Paz: AGRUCO, Plural Editores.

Díaz, C. J., Ojeda, R. M. y Valderrábano, P. D. (2016). Metodología de muestreo de poblaciones finitas para aplicaciones en encuestas. Veracruz: Imaginarial Editores.

Douwe, P. J. van der (2013). Diez cualidades de la agricultura familiar. Agricultura familiar campesina. CEISA Revista de Agroecología, 29(4), 6-7

FAO. (2012a). Estudios sobre innovación en la agricultura familiar. Experienciasy enfoques de procesosparticipativos de innovación en agricultura. Roma: FAO.

FAO. (2012b). Agricultura familiar con potencial productivo en México. Organización de las Naciones Unidaspara la Alimentación y la Agricultura. FAO-SAGARPA. Disponible en http://www.sagarpa.gob.mx/programas2/ evaluacionesExternas/Lists/Otros\%20Estudios/Attachments/42/Agricultura\%20Familiar_Final.pdf

Formichella, M. (2005). La evolución del concepto de innovación y su relación con el desarrollo. Estación Experimental Agropecuaria Integrada Barrow (Convenio MAAyP-INTA), Tres Arroyos. Disponible en https:// www.google.com.mx/search?q=Formichela + M. + la + evolucion + del + concepto + de + innovacion\&ie $=u-$ tf-8\&oe=utf-8\&client=firefox-b-ab\&gfe_rd=cr\&ei=NOn_V9qQJMa1 mQGhtZawDQ

French, J., Montiel, K. y Palmieri, V. (2014). La innovación en la agricultura: un proceso clave para el desarrollo sostenible. Costa Rica: Instituto Interamericano de Cooperación para la Agricultura. IICA-OEA. 
Hernández, S. R., Fernández, C. y Baptista, P. (2014). Metodología de la investigación. México: McGraw-Hill.

Herrera, T. F. (2013). Enfoques y políticas de desarrollo rural en México. Una revisión de su construcción institucional. Gestión y politica pública, 22(1), 131-159.

Kulshreshtha, A. D. (2013). Basic concepts of Sampling. Brief review: Sampling designs. U. N. Statistical Institute for Asia and Pacific. Retrevied from http://www.unsiap.or.jp/e-learning/el_material/Agri/rap2/ ag_02_04_L2.1_SamplingDesigns.pdf

Levin, R., Rubin, D., Balderas, M., Del Valle, J. y Gómez, R. (2004). Estadística para administración y economía. Séptima edición. México: Pearson Educación.

Montoya, S. O. (2004). Schumpeter, innovación y determinismo tecnológico. Scientia et Technica, 10(25), 209-213.

Morales, M., Ortiz, C. y Arias, M. (2014). Factores determinantes de la innovación: una mirada a la situación en Latinoamérica. Revista Escuela Administración de Negocios, 148-163. Recuperado de http://www.scielo. org.co/pdf/ean/n72/n72a10.pdf (Consultado 06-05-2017)

OCDE. (2005). Manual de Oslo. Guía para la recogida e interpretación de datos sobre innovación. Organización de cooperación y desarrollo económicos. Grupo Tragsa.

ONU. (2014). Conferencia de las Naciones Unidas sobre Comercio y Desarrollo. Instrumento de políticas de innovación para un desarrollo inclusivo. Ginebra: Naciones Unidas.

Pérez, L. C. (2010). El sistema estadistico SAS. Madrid: Gaceta.

Quintero, L. y Cortés, C. (2011). Cultura innovadora. Estudios de caso: sociología de las Pymes en Colombia. Bogotá: Universidad Nacional de Colombia.

Rendón, M. R. y Aguilar, A. J. (2013). Gestión de redes de innovación en zonas rurales marginadas. México: Porrúa.

Rodríguez, H. R., Cadena, I. P., Morales, M., Jácome, M. S., Góngora, G., Bravo, E., Contreras, R. (2013). Competitividad de las unidades de producción rural en Santo Domingo Teojomulco y San Jacinto Tlacotepec, Sierra Sur, Oaxaca, México. Revista Agricultura Sociedad y Desarrollo, 10(1), 111-126

Rojas, S. R. (2013). Guia para realizar investigaciones sociales. México: Plaza y Valdés Editores.

Rugeles, L., Guaitero, D., Saavedra D., Ariza, C., Noreña, H., Betancur, I. y Vargas, M. (2013). Medición de la innovación agropecuaria en Colombia. Medellín: Universidad de Medellín. Disponible en http://avalon. utadeo.edu.co/servicios/ebooks/innovacion_agropecuaria/\#4/z

Rugeles, L. (2014). Metodología paramedición deinnovación agropecuaria en Colombia. Encuesta de Innovación, Indice de Innovación y Modelo Econométrico. Bogotá: Universidad de Bogotá Jorge Tadeo Lozano. Red RAET de Universidades.

Scheaffer, R., Mendenhall, W.y Lyman, R. (2007). Elementos de muestreo. Sexta edición. Madrid: Thomson editores.

Sonnino, A. y Ruane, J. (2013). La innovación en agricultura familiar y las biotecnologías agrícolas como herramientas de las políticas de seguridad alimentaria. H. Hedson y T.Zamudio (eds.), Biotecnologías e innovación: el compromiso social de la ciencia. Bogotá: Pontificia Universidad Javeriana. Disponible en https://www. researchgate.net/publication/263443721_La_innovacion_en_agricultura_y_las_biotecnologias_agricolas_como_herramientas_de_las_politicas_de_seguridad_alimentaria (Consultado 15-01-2017)

Valencia, L. P. de y Patlan, J. (2011). El empresario innovador y su relación con el desarrollo económico. TEC Empresarial, 5(3), 21-27

Vargas, C. J., Palacios, R. M., Camacho, V. J., Aguilar, A. J. y Ocampo, L. J. (2015). Factores de innovación en agricultura protegida en la región de Tulancingo, México. Revista Mexicana de Ciencias Agrícolas, 6(4), 827-840

Villalobos, V. M., Chavarría, H., Campos, A., Salazar, E., Witkowki, K., Henriquez, P. y García, W. M. (2015). Implicaciones de las reformas a la PAC de la Unión Europea en el sector agrícola de América Latina y el Caribe. Ambienta. España. Disponible en http://www.iica.int/es/noticias-externas/implicaciones-de-las-reformas-la-pac-de-la-unión-europea-en-el-sector-agrícola-demericalatina

\section{BY-NC-ND}

